\title{
Registration and Fusion of Segmented Left Atrium CT Images with CARTO Electrical Maps for the Ablative Treatment of Atrial Fibrillation
}

\author{
G Nollo ${ }^{1}$, A Cristoforetti ${ }^{1}$, L Faes ${ }^{1}$, M Centonze $^{3}$, \\ M Del Greco ${ }^{2}$, R Antolini ${ }^{1}$, F Ravelli $^{1}$ \\ ${ }^{1}$ Department of Physics, University of Trento, Italy \\ ${ }^{2}$ Cardiology Division, S Chiara Hospital Trento, Italy \\ ${ }^{3}$ Radiology Division, S Chiara Hospital Trento, Italy
}

\begin{abstract}
This study aims to extract the interior surface of the left atrium (LA) and pulmonary veins ( $P V s)$ from threedimensional tomographic data and to integrate it with LA CARTO electrical maps. The separation of LA and PVs from other overlapping structures of the heart was performed processing 3D CT data by markercontrolled watershed segmentation and surface extraction. CARTO maps were then registered on the $L A$ internal surface by a stochastic optimization algorithm based on simulated annealing. The residual registration error resulted inferior to $3 \mathrm{~mm}$. The integration between electrophysiological and high resolved anatomic information of LA results feasible and may constitute a significant support for mechanism investigation and treatment of atrial fibrillation.
\end{abstract}

\section{Introduction}

An anatomical approach for radio-frequency (RF) ablation of paroxysmal atrial fibrillation (AF) is gaining increasing importance since the introduction of circumferential pulmonary veins (PV) isolation [1]. In this view, correlation of electrical patterns and ablative sites with the real anatomic structure of the atria may constitute an important challenge. The integration of left atrium (LA) electrophysiological information from CARTO maps (Biosense Webster), which exhibit poor geometrical details, with the more spatially resolved anatomical information obtained from tomographic imaging seems useful in order both to increase intervention success and to investigate source and maintenance of AF [2]. Goal of this work is the integration of the electrophysiological values obtained through CARTO on a high resolution LA reconstruction, produced from segmentation of the CT volume data.

\section{Methods}

\subsection{Data acquisition and preprocessing}

This procedure has been developed and tested off line on cases of paroxysmal AF referred for RF catheter ablation by a nonfluoroscopic navigation system (CARTO, Biosense Webster). 3D tomographic data were acquired prior to intervention with 16-slices spiral computed tomography (MX 8000 IDT, Philips; $0.75 \mathrm{~mm}$ collimation, iodinated contrast, retrospective ECG-gating at $75 \%$ of cardiac cycle). CT data were loaded in Matlab's programming software as almost isotropic 3D numerical matrix of Hounsfield units, each voxel standing for a cube with side length between $0.4-0.5 \mathrm{~mm}$. Memory requirements and reasonable computational time involved downsizing of the matrix by a half, setting the resolution to about $1 \mathrm{~mm}$. By means of a low-pass spatial filter resampling aliasing was prevented and noise reduced.

The CARTO navigation system is a minimally invasive apparatus for ablative treatment of cardiac arrhythmias that permits real time reconstruction and navigation of coarse electroanatomic maps from the interior wall of heart chambers. During the procedure, up to a few hundreds of the catheter tip locations are acquired sequentially, synchronized on the end diastolic phase. The electrogram is simultaneously recorded and analysed to produce salient electrophysiological information (peak to peak bipolar potentials, local activation time), linked to their surface position. From this set of points a low resolution 3D shape of the investigated cardiac chamber is reconstructed, showing electrical values in pseudocolour map. Their spatial coordinate along with corresponding electrical information were exported and fed to Matlab processing platform.

The extraction of the inner wall of cardiac chambers 
and connected vascular structures from CT data, after the preliminary filtering and downsizing, was performed by marker controlled watershed segmentation.

\subsection{Segmentation}

Among the several existing segmentation procedures the watershed transform (WT) is prominent owing to its ability of producing closed edges and separated regions in relatively brief computational time [3-5]. If we regard a grayscale bidimensional image as a topographic relief, each region obtained by this partitioning constitutes a catchment basin of the relief, that is all pixels from which the steepest slope path ends to the same regional minimum. The watershed lines are located on the crest between different basins, their pixels being the starting point of diverging descending paths reaching different minima. Because objects boundaries are to be related to high local intensity variations, a threedimensional extension of WT was applied actually to the gradient magnitude image. Since every local minimum is linked to a watershed region, on account of the great number of minima yielded by noise, the output of a direct WT is prejudiced by oversegmentation.

In order to overcome this drawback we resorted a marker controlled WT approach: the gradient magnitude image undergoes an homotopy transformation, finalized to force predefined areas (markers) as the sole local minima [6]. This operation may be better depicted through a flooding comparison: the elevation surface is pierced correspondingly to the markers points and water is rained upon until every sunken area is filled up. Thus every valley having no marker within becomes a plateau, levelled at the nearest marker height and connected to it, forming hence a unique regional minimum. Almost every marker produces one basin on the modified gradient image, which is detected finally by WT. It is evident that the crest line between two markers surviving the homotopy transformation is the one along which the minimum value is higher than the minimum values of all other competing crest lines. The watershed algorithm employed is based on the immersion simulation designed by Vincent and Soille [5].

About 20 - 30 markers were placed manually inside cardiac chambers and vases, scanning few transversal slices of the tomographic image, each labelled according to 6 different classes (LA, right atrium, left ventricle, right ventricle, aorta, pulmonary artery) under operator's knowledge and interpretation.

An ulterior background class, i.e. not opacified tissue and structures external to heart, was seeded through thresholding at level between $100-200 \mathrm{HU}$, depending upon opacification quality, and manually in high density osseous tissue difficult to reject in segmentation.

The gradient magnitude image was then produced via a
$3 \times 3 \times 3$ box Sobel-like kernel applied to the native intensity image, and subjected to homotopic transformation under the binary markers image. After that, the WT extracted the influence region of every disjointed marker area, which was classified by the marker label.

The result of this stage was a complete voxel classification allowing to isolate the inner volume of a single chamber, i.e. the left atrium. The atrial surface was then extracted from the binary image of left atrium by isosurfacing at threshold of 0.5 . The obtained mesh was finally resized to comply with isotropy requirements.

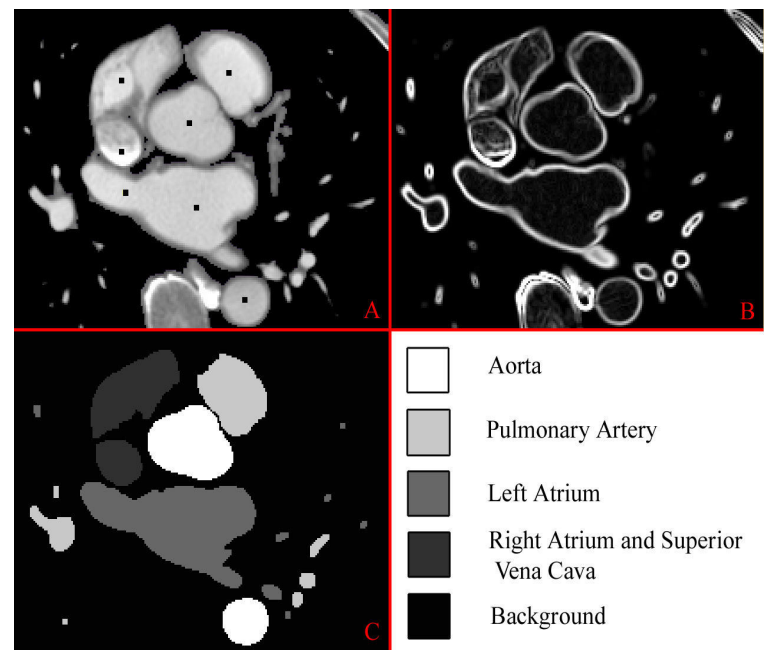

Figure 1. Same cross section of CT image during progressive stages of segmentation. A: Native CT transversal image where the background and the manual markers are superimposed in black. B: Gradient magnitude image at the same slice. C: Final watershed classification. Shades correspond to cardiac district listed in the legend of the lower right panel.

\subsection{Registration}

The integration comprises a registration step, whereby the multimodal data are spatially aligned, and a fusion step for the interpolation and visualization of the associated information [7,8], in this case the electrical values of the aligned CARTO points on the LA mesh. The registration process consisted in applying a spatial transformation to the CARTO points, trying to align them with the LA surface by minimizing a matching error (ME) between the two data sets.

The designed transformation was rigid, i.e. translation by a vector (three parameters) plus free rotation (three angles: roll, yaw and pitch); thus any loss of geometrical information due to deformation was avoided. We chose the square mean of distances of the points from the surface as quantification of mismatch. This value, as 
function of the six parameters of the rigid transformation, constituted the ME to minimize. The calculation of ME was implemented using a three-dimensional quasieuclidean distance transform image [9], preliminarily produced from the segmented atrial surface. For any parameters combination, the position of the CARTO points in the distance image was computed and the corresponding ME immediately estimated. The adopted optimisation strategy made use of simulated annealing (SA) [10]. SA is a random reiterated search in the parameters space wherein every new search point is taken in the surroundings of the previous and is accepted with a probability dependent on the variation of the ME.

Three definition are then required:

1. The transition distribution function $G$ rules the change probability from configuation $p$ to a new candidate $q$ as function of their distance. We opted for a gaussian, where $\sigma$ is the step size:

$$
G(p, q)=c o s t \cdot \exp \left(-\frac{\|q-p\|^{2}}{2 \sigma^{2}}\right)
$$

2. The acceptance probability $A$ of new candidates depends on $\mathrm{ME}$ variation and is tuned by a parameter $\boldsymbol{t}$, which is changed during the process. We used a Boltzmann-like acceptance:

$$
A(p, q, t)=\min \left[1, \exp \left(-\frac{M E(q)-M E(p)}{t}\right)\right]
$$

3. An appropriate schedule for $\boldsymbol{t}$.

This algorithm differs from hill descent because the climbing steps have a nonzero acceptance probability, preventing thus trapping into local minima. For the parallelism to the physical system from which this algorithm originated (condensed matter physics) [11], $\boldsymbol{t}$ is often called temperature. If temperature schedule is decreased at a sufficiently low rate the system has high chance to freeze in a global minimum state.

The step size $\sigma$ has been varied during the cooling to match the temperature, so that acceptance rate were ranging from about 0.4 to 0.6 , giving maximal efficiency.

\section{Results}

\subsection{Segmentation}

As can be seen in fig. 2, the result of segmentation was accurate and capable of tracing the vascular ducts down to $2 \mathrm{~mm}$ thin. The morphology of both posterior and anterior side of the atrium, of the ostial junction and of pulmonary veins branching tree is delineated in detail, receiving a qualitative validation through comparison with native sectional images and expertise by the radiologist.

\subsection{Registration}

An example of registration result is shown in fig. 3, where the misaligned CARTO point have been brought to a matching position on the atrial surface with a residual square mean distance between points and surface of 2.4 $\mathrm{mm}$. It should be however remarked that the real error between homologous points of CT and CARTO is expected to be larger.

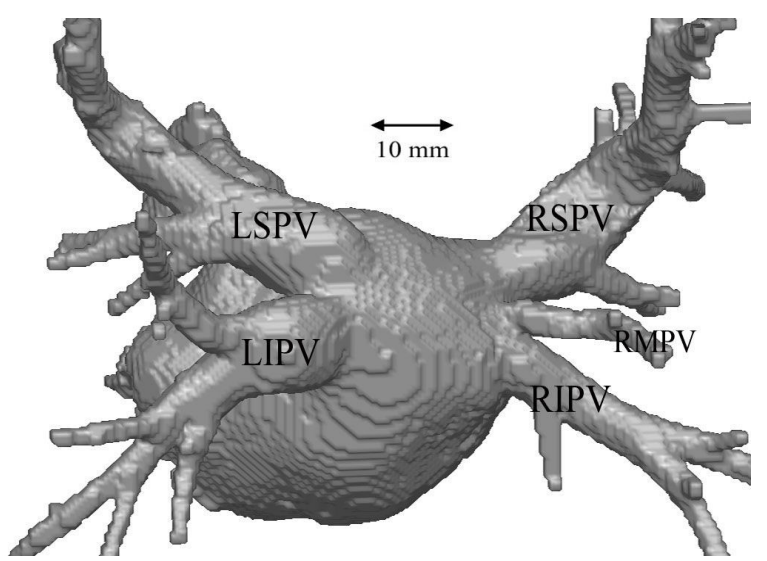

Figure 2. LA inner surface extracted from CT data by watershed segmentation and isosurfacing (LSPV, LIPV, RSPV, RMPV, RIPV: left superior and inferior, right superior, middle and inferior pulmonary veins respectively).

To verify the actual problem solving capability of the procedure, the final states of repeated cooling from several different starting configuration were compared. Above 10000 iterations the configurations settled down to a scattering range of about $1 \mathrm{~mm}$ around a unique configuration. On a statistical basis, this configuration has to be considered the global minimum on the matching error. Seeing that the estimated input localization error between CT and CARTO data is around $3 \mathrm{~mm}$ at least, further optimisation precision was meaningless.

\section{Discussion and conclusions}

A non parametric, semiautomatic segmentation method grounded on marker controlled watershed transform has been developed and successfully applied to separate inner walls of cardiac chambers from contrast enhanced CT images. The segmentation of left atrium is of particular concern to planning ablative treatment, inasmuch it allows the morphological characterisation of $\mathrm{PV}$ and its ostia. In general this technique proves to be useful for visualization, morphology analysis and volume 
quantification of all the cardiac districts.

Contrary to other previous attempts of online alignment [12], our registration procedure of high resolution atrial surface with the electrical CARTO map has been achieved without any external or internal preparatory reference device, but using only the geometric information of the data. Therefore it is a completely retrospective procedure, which rely uniquely on shape matching. Although so far it has been performed offline, in the future it could be developed for a real time application. By means of a stochastic relaxation approach we obtained a registration precision inferior to $1 \mathrm{~mm}$ and a residual error inferior to $3 \mathrm{~mm}$. The CARTO points localisation on the atrial surface allows the fusion of the electrical information utilizing one of the several 3D points interpolation algorithm [13], providing a useful tool for intervention validation, for investigation of linkage between stenosis and ablation scheme, for studying of mechanisms underlying generation and maintenance of fibrillation.

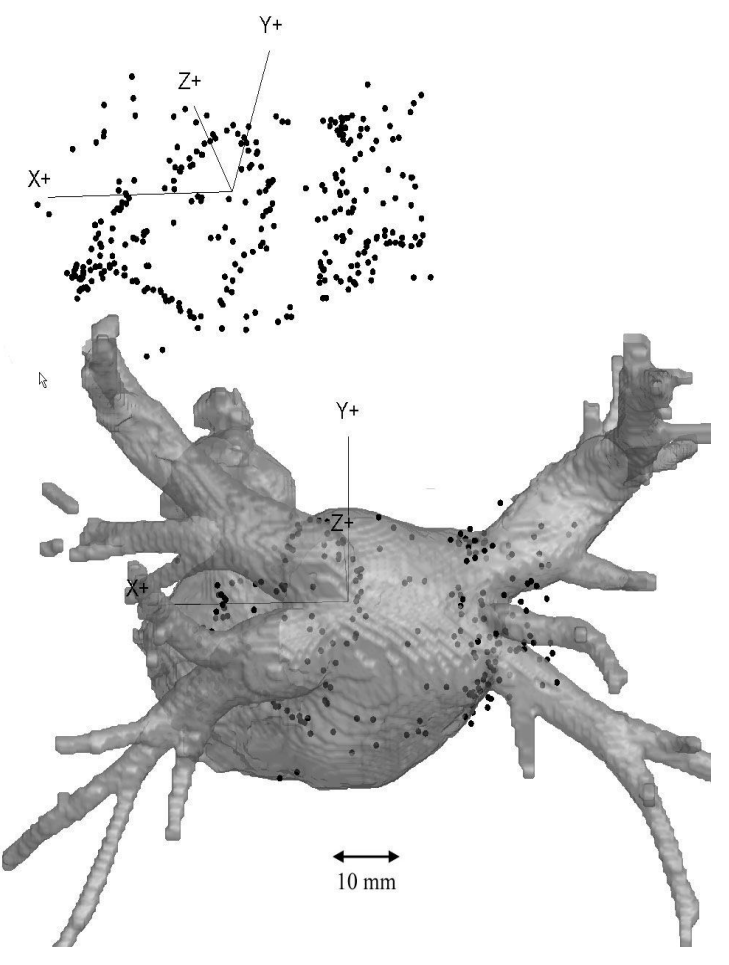

Figure 3. Up: CARTO points. Down: surface rendering of LA inner surface, on which the CARTO points has been registered. The surface is semitransparent to make visible the points placed just inside.

\section{References}

[1] Pappone C, Rosanio S, Oreto G et al. Circumferential radiofrequency ablation of pulmonary vein ostia: A new anatomic approach for curing atrial fibrillation. Circulation 21-11-2000;102[21]:2619-2628.

[2] Packer D L Evolution of mapping and anatomic imaging of cardiac arrhythmias. Pacing Clin.Electrophysiol. 2004;27[7]:1026-1049.

[3] Beucher S. The watershed transformation applied to image segmentation. Cambridge, UK, Scanning Microscopy International,. 1992; suppl. 6:299-314.

[4] Beucher S, Lantuejoul C. Use of watershed in contour detection. International Workshop on Image Processing: Real Time Edge and Motion Detection/estimation IRISA 1979;132:2.1-2.12.

[5] Vincent L, Soille P. Watershed in digital spaces: an efficent algorithm based on immersion simulations. IEEE Trans.Pattern Anal.Mach.Intell. 1991;13[6]:583-598.

[6] Vincent L. Morphological grayscale recostruction in image analysis: Applications and efficient algorithms. IEEE Transaction on Image Processing 1993;2[2]:176-201.

[7] Matsopoulos G K, Delibasis K K, Mouravianskly N A. Medical image registration and fusion techniques: $A$ review. Stergiopulos, S. Advanced Signal Processing Handbook: Theory and Implementation for Radar, Sonar, and Medical Imaging Real Time Systems. 2000.

[8] Maintz J B A, Viergever M A. A survey of medical image registration. Medical Image Analysis 1998;2[1]:1-36

[9] Paglieroni D. Distance Transforms: Properties and Machine Vision Applications. ComputerVision, Graphics, and Image Processing: Graphical Models and Image Processing 1992;54[1]:57-58.

[10] Laarhoven P J M, Aarts E H L. Simulated Annealing: Theory and Application. 1987. D. Riedel Publishing Company.

[11] Metropolis N, Rosenbluth A W, Rosenbluth M N et al. Equation of state by fast computing machines. The Journal of Chemical Physics 1953;21[6]:1087-1092

[12] Solomon S B, Dickfeld T, Calkins H. Real-time cardiac catheter navigation on three-dimensional $\mathrm{CT}$ images. J.Interv.Card Electrophysiol. 2003;8[1]:27-36.

[13] Amidror I. Scattered data interpolation methods for electronic imaging systems: a survey. Journal of Electronic Imaging 2002;12[2]:157-176.

Address for correspondence

Giandomenico Nollo.

Laboratorio di biosegnali, Dipartimento di Fisica, Università degli Studi di Trento

Via Sommarive 14, 38050 Povo (TN), Italy

nollo@science.unitn.it 